\title{
UN ACERCAMIENTO AL TRABAJO COLABORATIVO
}

Patricia Elízabeth Glinz Férez

\section{INTRODUCCIÓN}

El avance tecnológico y el mundo globalizado, resultantes de movimientos sociales, científicos, industriales y filosóficos, modificaron las formas de existencia de los seres humanos contemporáneos. A partir de la década de los setenta del siglo pasado el Estado mexicano inició una reforma educativa que respondía a las necesidades del momento, misma que ha seguido evolucionando y transformándose según lo exige el sistema; en algunos casos lo ha hecho de manera satisfactoria y en otros con carencias palpables y difíciles de superar.

Las dificultades que han surgido del proyecto tradicional de aprendizaje, las que se sustentan en la retención y reproducción de conceptos, ideas y hábitos de las sociedades, no son resultado del progreso de investigaciones o de la ciencia, tampoco lo son de las teorías de psicología; germinan a partir de la mezcla de los cambios que la tecnología ha conseguido, de los movimientos sociales acontecidos, así como de los efectos culturales desencadenados por la búsqueda incesante del progreso.

Los pensadores posmodernos aplicando la hermenéutica, en lo que respecta al avance de los pueblos, han apostado por la educación como el medio eficaz para œnducirlos a la construcción de un mundo mejor: "frente a los numerosos desafíos del porvenir, la educación constituye un instrumento indispensable para que la humanidad pueda progresar hacia los ideales de paz, libertad y justicia social" (DELORS, 1997:9). Éste autor en su informe presentado a la UNESCO, prepondera como las características esenciales para la educación en el siglo XXI, cuatro premisas que todos los gobiernos mediante su sistema educativo, deben implantar: aprender a aprender, aprender a hacer, aprender a ser $y$ aprender a trabajar en grupo.

Las naciones podrán sustentar su avance en todos los rubros si logran diseñar y aplicar procesos que generen resultados a partir de la propuesta anterior. Las sociedades actuales, envueltas en la cantidad de información que es generada por los medios productivos y de comunicación, no pueden tener las mismas demandas de aprendizaje que en épocas anteriores. Un sólo tema puede ser escrito por cientos de autores, con visiones y propuestas diferentes; asimismo un individuo, puede gastar su vida entera, tratando de obtener y aprender dicha información sin poder concretar algo, puesto que memorizar sería su principal barrera.

Para desarrollar las habilidades propuestas por la UNESCO, sólo existe el camino del aprendizaje constructivo, mismo que apoyará el despliegue de habilidades cognitivas en los alumnos. ¿Cómo propiciar cambios de estructuras mentales cognoscentes en los estudiantes de cualquier grado escolar? ¿Cómo conducir al alumno hacia el trabajo en equipo? ¿Cómo enseñar a aprender? Son algunas de las incógnitas que surgen en la mayoría de los docentes, y que sólo ellos pueden responder, cuando realmente se involucran en el proceso enseñanza-aprendizaje, desde su inicio, hasta la culminación del mismo y de la propia retroalimentación. 
Para el logro de cualquier objetivo, que se plantee como meta en una clase, un periodo determinado o un ciclo escolar, deberán los maestros aplicar las llamadas estrategias de aprendizaje. "Lo que la escuela y el maestro puedan lograr por medio de su labor será mínimo si no organizan e integran claramente objetivos, actividades y recursos dentro del marco de una planeación didáctica" (MORENO, 1994:90). Son un conjunto de actividades, técnicas y medios que se planean, en concordancia con las necesidades de la población estudiantil o social a la que se dirigen, los objetivos que persiguen y la naturaleza de las áreas y cursos, con el fin de lograr mayor efectividad en el proceso de aprendizaje.

"Las estrategias de aprendizaje constituyen el aprendizaje de los procedimientos de autocontrol y autorregulación cognitiva sobre la atención, la memoria y la comprensión" (VILLALOBOS, 2002:221). Es importante puntualizar que sumadas a los contenidos, los objetivos y a la evaluación de los aprendizajes, forman los elementos fundamentales del proceso de aprendizaje.

Un alumno utiliza una estrategia, cuando le es posible ajustar su comportamiento, (lo que piensa y hace), a los requerimientos de una actividad o tarea que debe efectuar, y a las circunstancias en que se realiza. La actuación del estudiante es estratégica cuando:

- Reflexiona conscientemente sobre el objetivo de la tarea.

- Planifica lo que va a realizar y cómo lo ejecutará.

- Realiza la tarea o proyecto.

- Evalúa su desempeño.

\section{TRABAJO GRUPAL}

La educación en la actualidad requiere del trabajo de grupo. En las actividades de enseñanza aprendizaje, el trabajo colaborativo o cooperativo (términos utilizados indistintamente) conforma uno de los principales elementos. Los proyectos innovadores que usan técnicas de enseñanza aprendizaje involucran esta modalidad de trabajo en la que el ser que aprende se forma como persona.

En el proceso de enseñanza tradicional, el aprendizaje se ha tomado como la transmisión de conocimientos; en los últimos tiempos el paradigma ha cambiado sustentado en la transferencia de conocimientos. Los procesos que los alumnos utilizan son de adecuación, como se plantea el constructivismo. La comunicación con los pares abre la percepción de la persona, desarrolla habilidades cognitivas y de trabajo en grupo, respondiendo a las necesidades que se conciben para esta época.

\section{APRENDIZAJE COLABORATIVO}

Esta técnica se refiere a la actividad que efectúan pequeños grupos de alumnos dentro de las aulas de clase; éstos se forman después de las indicaciones explicadas por el docente. Durante el inicio de la actividad y al interior del grupo, los integrantes intercambian información, tanto la que activan (conocimientos previos), como la que investigan. Posteriormente trabajan en la tarea propuesta hasta que han concluido y comprendido a fondo todos los conceptos de la temática abordada, aprendiendo así a través de la cooperación.

Si se comparan los efectos que se logran al aplicar esta técnica, contra la tradicional, se puede afirmar que los alumnos aprenden de manera significativa los contenidos, desarrollan habilidades cognitivas 
(razonamientos, observación, análisis, juicio crítico, etc.), socializan, toman seguridad, se sienten más aceptados por ellos mismos y por la comunidad en que se desenvuelve.

Las tres estructuras que forman el trabajo colaborativo son: la competencia, mediante la cual los alumnos tratan de alcanzar las metas, mismas que sólo se consiguen cuando el grupo en su totalidad lo hace, (si yo gano tu ganas), por medio de la cooperación, los alumnos ejercitan la interdependencia positiva, logran un crecimiento personal y social. El individualismo a diferencia de la primera, proporciona solamente un crecimiento individual o personal, pero el alumno tiende al aislamiento, lo que le puede provocar daños permanentes en su interioridad.

Los cinco componentes del aprendizaje cooperativo, son "la interdependencia positiva, la responsabilidad individual, la interacción fomentadora cara a cara, las habilidades interpersonales, y el procesamiento por el grupo" (FUENTES, 2003).

\section{EL AULA DURANTE EL TRABAJO COOPERATIVO}

Se convierten en espacios de expresión oral y comunicación, entre compañeros estudiantes, así como entre los profesores y alumnos. Las actividades son dispuestas, para que los alumnos expongan y compartan sus ideas acerca del tema en estudio al interior del equipo, lo que investigan y aprenden; pueden jugar diferentes roles como: Abogado del diablo, secretario, supervisor, motivador, administrador de materiales, observador, reportero, controlador del tiempo. Pueden intercambiarlos si sienten ser mejores para otro papel; existe una permutación de ideas y se apoyan mutuamente. Los resultados serán del trabajo grupal, no del individual.

\section{ELEMENTOS DEL APRENDIZAJE COOPERATIVO}

COOPERACIÓN. Los alumnos se apoyan entre ellos para adquirir firmemente los conocimientos de la temática en estudio. Además de desarrollar habilidades de trabajo en equipo (socialización), comparten todos los recursos, logros, metas. El éxito individual, depende del éxito del equipo.

RESPONSABILIDAD. Los alumnos son responsables del porcentaje del trabajo que les fue asignado por el grupo. Pero el grupo debe permanecer involucrado en la tarea de cada uno de los integrantes y se apoyan en los momentos de dificultades.

COMUNICACIÓN. Exponen y comparten la información recabada relevante, se apoyan en forma eficiente y efectiva, se retroalimentan para optimizar su trabajo, analizan las conclusiones de cada integrante y por medio de la reflexión buscan obtener resultados de mejor calidad.

TRABAJO EN EQUIPO. Los alumnos aprenden juntos a resolver la problemática que se les presenta, desarrollando habilidades de comunicación, liderazgo, confianza, resolución de problemas y toma de medidas hacia un problema.

AUTOEVALUACIÓN. Cada grupo debe evaluar su desempeño, tanto sus aciertos como sus errores, para enmendarlos en la siguiente tarea a resolver. El equipo se fija las metas y se mantiene en continua evaluación para rectificar los posibles cambios en las dinámicas con la finalidad de lograr los objetivos. 


\title{
6. TIPOS DE APRENDIZAJE COOPERATIVO
}

\begin{abstract}
"Para que un grupo sea cooperativo, en el grupo debe existir una interdependencia positiva bien definida y los integrantes tienen que fomentar el aprendizaje y éxito de cada uno cara a cara, hacer que todos y cada uno sea individual y personalmente responsable por su parte equitativa de la carga de trabajo, usar habilidades interpersonales y en grupos pequeños correctamente y recapacitar (o procesar) cuán eficaz es su trabajo colectivo. Estos cinco componentes esenciales hacen que el aprendizaje en grupos pequeños sea realmente cooperativo" (JOHNSON, et al, 1995:36).
\end{abstract}

INFORMAL

"El aprendizaje cooperativo informal consiste en exigir que los estudiantes trabajen juntos para lograr una meta de aprendizaje colectiva en grupos temporales especiales que duran desde unos cuantos minutos hasta todo un período de clase" (JOHNSON, et al, 1995:51). Este tipo es utilizado cuando el docente requiere que el alumnado, dirija sus habilidades hacia un tema determinando, y a conceptos que deben aprender; asimismo para lograr que se fomente el aprendizaje, dentro de un ambiente cordial al interior de los equipos, "para ayudar a organizar por adelantado el material que se abarcará en una sesión de clases, para garantizar que los estudiantes procesen cognitivamente el material que se está impartiendo y para proporcionar una conclusión a la sesión didáctica" (Ibid).

Este tipo de grupos avalan la identificación y corrección de errores, falta de comprensión de algunos conceptos o relaciones. Propician el aprendizaje personalizado. Asimismo pueden aplicarse en todo momento del proceso, pero son significativamente útiles, cuando el docente requiere hacer una exposición oral por la complejidad que represente la temática, o para la instrucción vertical. Garantiza que la información realmente sea procesada por el alumno, y no se conviertan en una colección de apuntes copiados del pizarrón durante la exposición del maestro. Mantiene a los estudiantes activos intelectualmente y el interés por la temática puede resultar motivador, propiciando que de manera personal y fuera del ámbito escolar se profundice en la temática en estudio.

\section{FORMAL}

"En los grupos de aprendizaje convencional, los estudiantes trabajan juntos durante períodos que abarcan desde una hora de clase hasta varias semanas para lograr objetivos comunes de aprendizaje y completar tareas y asignaturas específicas" (JOHNSON, et al, 1995:38). Estos grupos formales, se pueden utilizar en diferentes formas, con finalidades siempre encaminadas a la adquisición de conocimientos, para adquirir nuevos conceptos, solucionar problemas, experimentos científicos, redacciones, etc.

El docente, aporta durante el proceso la enseñanza de conceptos, tipos de estrategias o métodos que facilitan el aprendizaje, observa de cerca la evolución y aprovechamiento de los grupos de trabajo, apoya a los alumnos cuando éstos lo necesitan. Evalúa el aprovechamiento del alumnado, según los criterios estipulados previamente, y "garantiza que los grupos procesen cuán eficazmente trabajaron juntos los integrantes" (lbid). Los estudiantes piden apoyo al profesor, a sus compañeros, buscan la retroalimentación, para tener elementos que les evite cometer los mismos errores y aplicar los aciertos en futuros trabajos.

El maestro desempeña un papel de seis etapas en el aprendizaje cooperativo (Jonson y Jonson 1994; Jonson, Jonson y Holubec 1993): 
1. Especificar los objetivos de la lección. Debe explicar claramente antes del inicio de la instrucción dos tipos de objetivos; el académico, el cual corresponderá al grado o nivel de los estudiantes; y el de habilidades sociales.

2. Tomar decisiones previas a la instrucción acerca de los grupos de aprendizaje, la distribución física del salón, los materiales didácticos y las funciones desempeñadas por los estudiantes dentro del grupo. Los grupos deberán conformarse de preferencia en binas y con un máximo de cuatro integrantes, siendo la tarea la que en realidad determine ese número. El tiempo es un factor importante, entre menor sea el tiempo el grupo deberá ser más reducido. Existen diferentes estrategias para formar los grupos, mismas que el maestro debe conocer.

Organización física del salón. Debe ser ordenado de manera que el profesor pueda transitar con facilidad, pero sobretodo que los alumnos puedan trabajar cara a cara, que se encuentren lo suficientemente cerca para dialogar en voz baja y armónicamente.

Selección de materiales didácticos. El maestro dependiendo del grado de madurez y habilidad de los alumnos distribuirá y asignará los materiales dentro del grupo o dejará de hacerlo cuando los integrantes tengan altos niveles de interdependencia personal.

Asignar funciones para garantizar la interdependencia. El maestro al planear la sesión debe de visualizar las funciones que desempeñarán los alumnos, pero cabe mencionar que entre ellos mismos, han reconocido las habilidades óptimas de cada uno, por lo cual les gusta jugar ese papel. El guía o habilitador deberá incitar a los integrantes de cada grupo para que se compruebe la comprensión del concepto 0 temática en cuestión, pueden encargarse todos de esta función o uno en específico.

3. Explicar la estructura de tarea y objetivos. Explicar la tarea académica. Primero el maestro explicará la tarea a realizar y que se espera obtener con ésta lección. Posteriormente, definirá los objetivos específicos de la lección, los cuales giran alrededor de la temática, debe activar los conocimientos previos, de manera que los alumnos tengan la oportunidad de traerlos a la memoria y enlacen la nueva información, con la finalidad de modificar las estructuras mentales, y propiciar el aprendizaje significativo.

"Cuando estén aclarados los procedimientos y objetivos, entonces puede tener lugar la enseñanz a directa de conceptos, principios y estrategias" (JOHNSON, 1995:43). También deben ser explicados con claridad los criterios de evaluación así como la conducta deseada o esperada por parte de los grupos durante el proceso, se incluyen aquí las expectativas de aprendizaje al interior del grupo (todos deben dominar el tema). Debe también incitar al grupo a participar activamente, aportando, cuestionando, y criticando (los conceptos, no a los compañeros). Los estudiantes deben tener funciones diferentes dentro del trabajo cooperativo, sin embargo es necesario que ejerciten en repetidas ocasiones el mismo papel, para que se dé un cambio de conducta y pueda ejecutarlo en forma natural.

1. Poner en marcha la lección cooperativa. El maestro debe configurar estructuras para que se dé la interdependencia positiva, y lograr por otro la responsabilidad individual. Existen guiones que han diseñado estudiosos de la materia, como el de Donald Dansereau, para estructurar la interdependencia de los estudiantes. Entre los más acreditados se encuentra el de MURDER, cuya traducción quiere decir: movilizar, entender, recordar, detectar, expandir, revisar); agrupados en binas, los alumnos se preguntan y corrigen mutuamente, hasta comprobar que se ha dado el aprendizaje. 
Existen otros tipos como el Co - op. Co - op, donde cada grupo tiene un minitema, por interés común comparten los conceptos, estructuran y planean conjuntamente. El rompecabezas, donde cada alumno estudia una parte, misma que expone ante el grupo, y el grupo conjuntamente hace su síntesis o resumen. El paquete de estudios TGT, donde realizan un torneo de equipos y juegos, el maestro realiza la exposición, y posteriormente se realizan concursos, cada equipo será heterogéneo, con un estudiante de nivel alto, otro de nivel medio y uno de bajo rendimiento. EI TAI (enseñanza asistida por el grupo), aplicado en equipos de cuatro o cinco personas, donde cada uno trabaja solo, comparten su experiencia de aprendizaje, se examinan entre ellos, comprueban el progreso; la evaluación es realizada por el profesor de manera individual. Otro tipo es CIRC (lectura y composición integradas y cooperativas), ésta técnica utiliza materiales que garantizan que el aprendizaje del grupo sea aplicado a la lectura; se implica durante el proceso la decodificación fónica y las habilidades de comprensión, además del significado, la evaluación es individual.

2. Vigilar eficacia de los grupos de aprendizaje cooperativo, intervenir cuando se requiera. El docente deberá mantenerse atento y observador a cualquier cambio de conducta que presenten los estudiantes, su progreso y desarrollo de habilidades, así como de los valores adquiridos; puede valerse para este tipo de seguimiento de un anecdotario, o instrumentos diseñados por él mismo. Su observación debe ser metódica, anecdótica y descriptiva; además de vigilar la sociabilidad de los alumnos, e intervenir cuando se requiera.

Evaluar el aprendizaje y la interacción de procesamiento. El profesor debe propiciar que al finalizar la lección se realice una conclusión del tema, debe intervenir al detectar carencias. Puede asimismo realizarla él. Se evaluará la calidad y cantidad de aprendizaje de los estudiantes, sin olvidar los criterios que se determinaron en cada caso o para el curso, debe recordar que evaluar no es calificar.

\section{GRUPOS BASE COOPERATIVOS}

Este tipo de grupos de aprendizaje cooperativo, está formado por "un número fijo de miembros cuyas responsabilidades principales son ayudar a los estudiantes a brindarse apoyo, estímulo y asistencia mutuos para completar tareas y compartir responsabilidad de esforzarse por aprender" (JOHNSON, 1995:55). Éste tipo de grupo es heterogéneo, se reúnen en forma periódica; su duración varía entre una sola clase, hasta un semestre o la carrera completa. Sus funciones son sobre todo de apoyo, tanto académico como social, entre las tareas que realiza se encuentran las rutinarias, las de apoyo personal. Estos grupos deben tener su identidad particular, para su fácil identificación, son un gran sostén para los integrantes del grupo, sobre todo porque todo lo que tiene que ver con la tarea, queda implícito. Otro beneficio que se obtiene al pertenecer en grupo de éste tipo, es que aprenden a sostener relaciones largas, perdurables, y mantienen un equilibrio entre los intereses personales y los del grupo.

Estos grupos pueden ser aprovechados e insertados en tareas de aprendizaje cooperativo formal e informal; mediante los cuales los alumnos fortalecen lo aprendido en clase, comprueban su apreciación y comprensión de los contenidos estudiados y de las tareas, además establecen lazos de amistad más fuertes con sus compañeros.

Las actividades de aprendizaje en grupos cooperativos, deben aplicarse a lo largo de cualquier curso. Tienen la bondad de propiciar el desarrollo de habilidades cognitivas en los alumnos, tales como: 
aprender a procesar la información, analizar, sintetizar, además de socializar, lo que conduce a la comprensión de que mediante el trabajo grupal los resultados que se obtienen, alcanzan mayor amplitud por la interacción cognitiva de los integrantes y aumenta la visión de la realidad de todo estudiante y profesionista.

Los individuos que lo practiquen en forma constante, desarrollarán su potencial, aprenderán a aprender en forma autónoma, adquirirán las habilidades y valores necesarios para enfrentarse al reto que representa la subsistencia del individuo de la época actual.

\section{METODOLOGÍA IMPLANTADA}

La actividad colaborativa informal tuvo los siguientes objetivos cognitivos:

Conocer, identificar y sintetizar, los cambios que se dieron en el estilo de vida, en lo tecnológico, científico, social y político, la situación que se vivió en el periodo entre guerras (1919-1939). La nueva revolución técnica y el bienestar social que propició. La situación de "Los felices años veintes", la bonanza de EUA; así como los avances tecnológicos y científicos que propiciaron tal efecto.

Objetivos colaborativos:

a) Compartir, escuchar, opiniones e ideas, mediante análisis de la exposición del profesor, compañeros y apoyo del texto, para enriquecer su propio punto de vista y propiciar el aprendizaje.

b) Relacionar los hechos de finales del siglo XIX, con los de principios del siglo XX.

c) Formular conclusiones de los acontecimientos más relevantes del periodo.

d) Construir un mapa conceptual, con los elementos que les proporcionó el maestro, mediante análisis grupal, determinen los cambios que se dieron en el estilo de vida, en lo tecnológico, científico y político.

\section{RESULTADOS DE ACTIVIDAD COLABORATIVA INFORMAL.}

Los alumnos trabajaron en binas, alcanzaron porcentajes entre 75 y $100 \%$ en la mayoría de los objetivos planteados, que se reflejaron en la rúbrica de reflexión y autoevaluación aplicada a los mismos. Los resultados obtenidos respecto a la primera actividad implantada fueron los que se muestran en las siguientes gráficas:

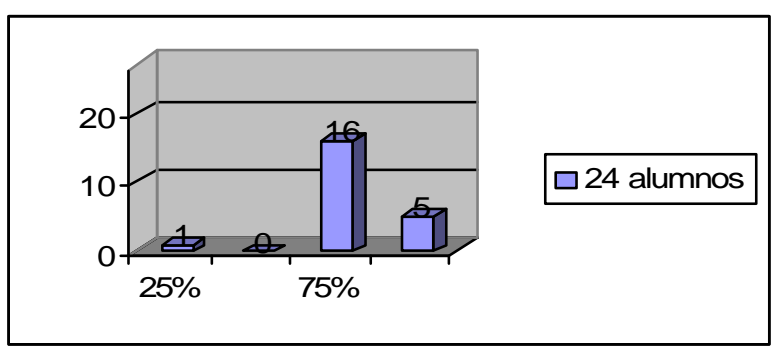

F.1a. Estuve atento a la explicación del maestro en un:

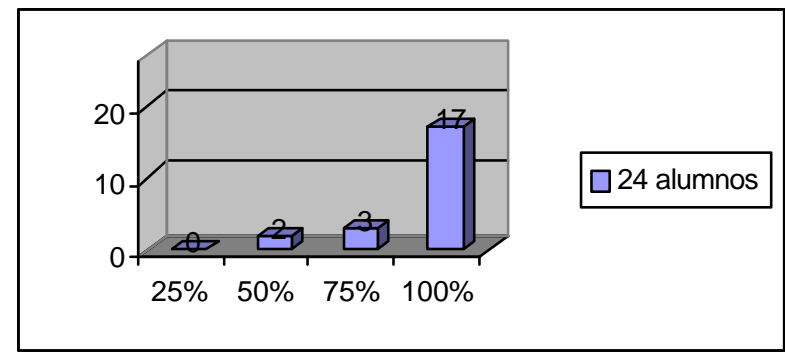

Fig. 2a. Valoré el trabajo de mi compañero en un: 


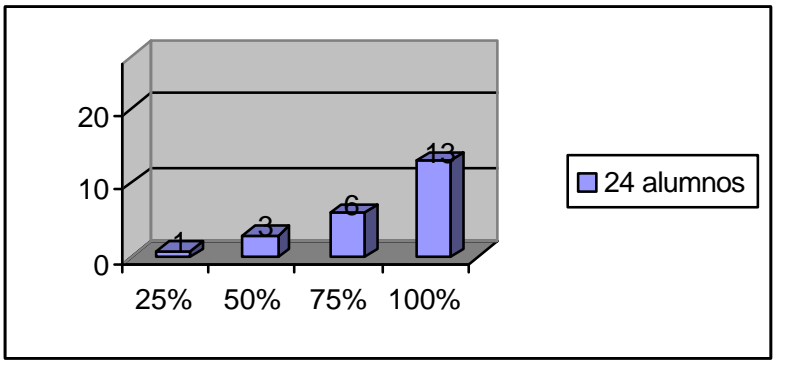

F.3a. Aporté ideas a mi compañero en un: aspectos positivos de mi compañero en un:

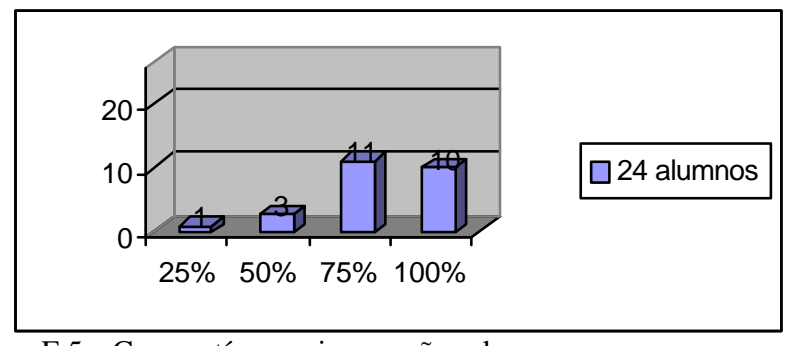

F.5a. Compartí con mi compañero la nueva información para resolver la tarea con mayor facilidad

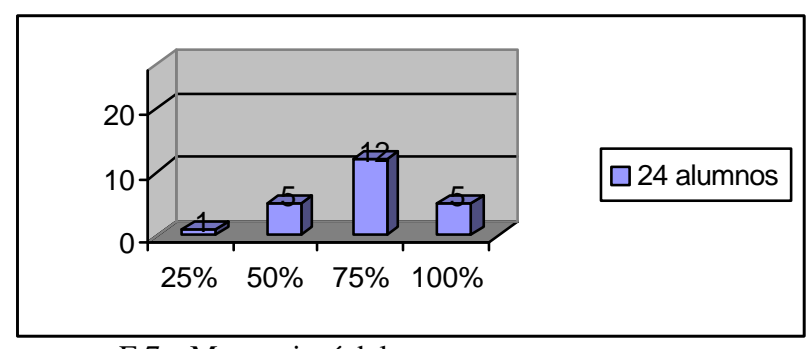

F.7a. Me cercioré del

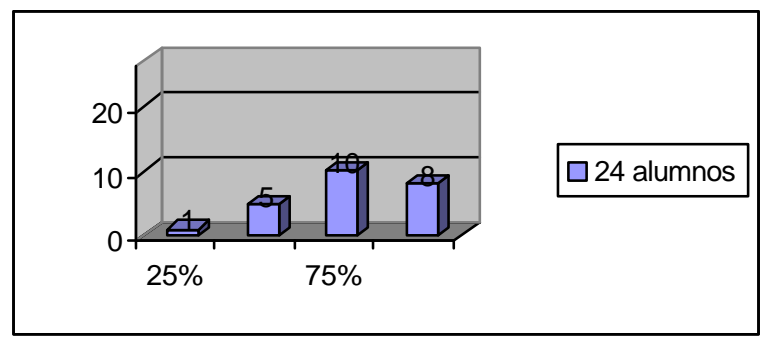

F.9a. La actividad despertó mi creatividad en

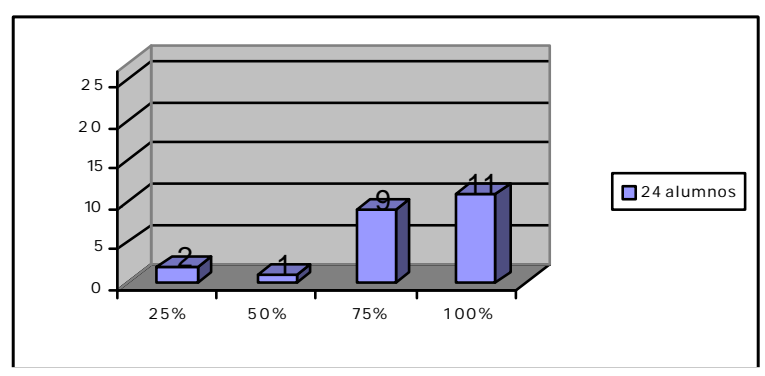

F.11a. Reforcé el valor de la responsabilidad en un:

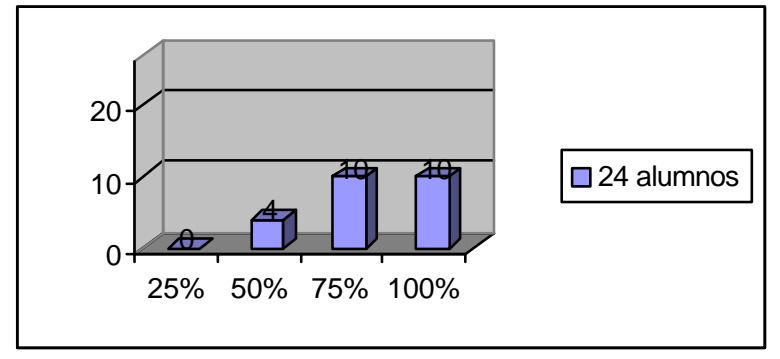

F.4a. Durante el trabajo colaborativo aclaramos las dudas surgidas del tema en un en

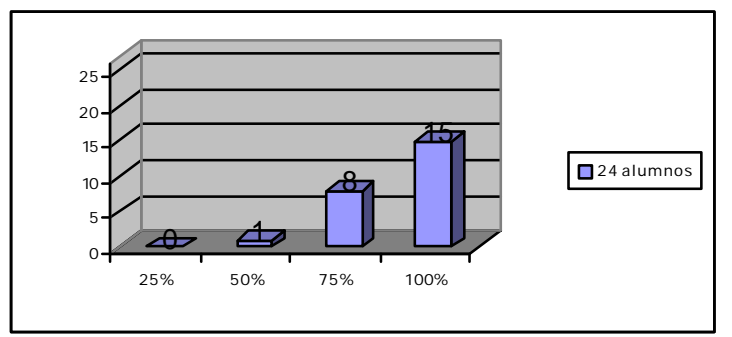

F.6a. El tema fue de fácil comprensión, porque elaboré con mi compañero un mapa conceptual en un:

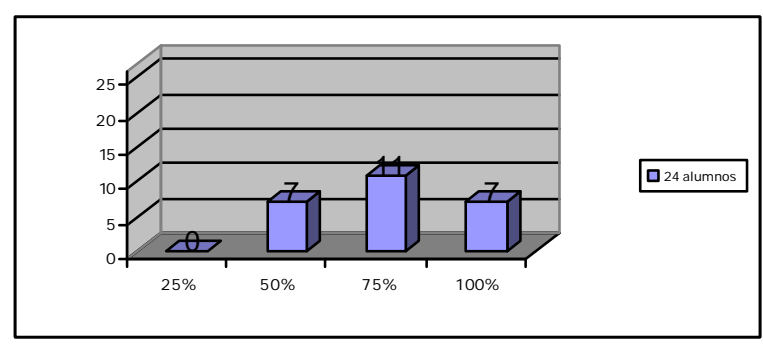

F.8a. Puedo explicar el tema sin dificultad en un:

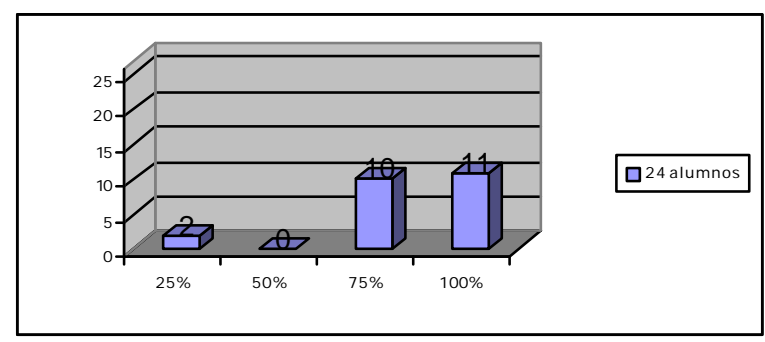

F.10a. El trabajo colaborativo me agrada en un:

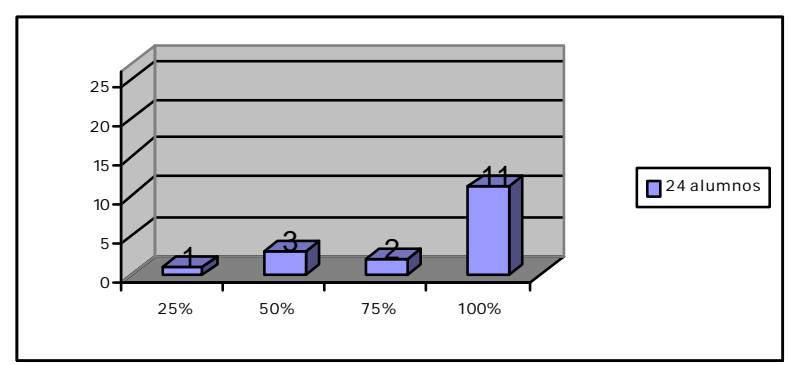

F.12a. Observé los aspectos positivos de mi compañero en un: 


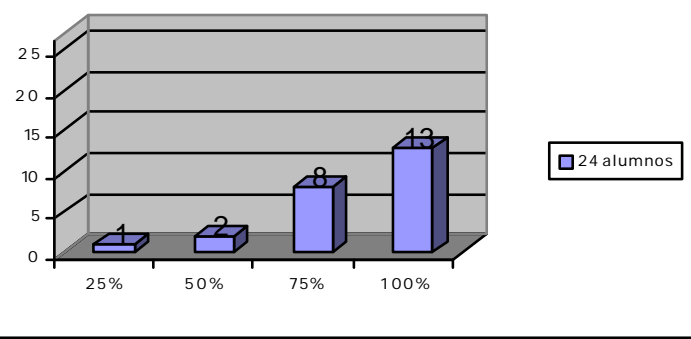

F.13a. Alcancé los objetivos fijados en un:

Para la realización de la actividad colaborativa formal se plantearon los siguientes objetivos cognitivos:

a) Conocer, identificar, analizar, procesar y sintetizar, los aspectos generales de la Segunda Guerra Mundial, así como las causas, consecuencias y su desarrollo cronológico.

Objetivos colaborativos:

b) Compartir, escuchar, y enriquecer el conocimiento mediante el intercambio de opiniones e ideas, análisis de la exposición del profesor, compañeros ya apoyo del texto.

c) Elaborar un diagrama "V" heurística, con la información contenida en su texto e investigación realizada previamente, mediante análisis grupal.

d) Comparar las dos Guerras Mundiales.

\section{RESULTADOS DE LA ACTIVIDAD COLABORATIVA FORMAL.}

Se formaron grupos de trabajo colaborativos de cuatro integrantes, cada uno con su rol bien definido. La actividad se realizó acorde a lo planeado, se cumplieron los objetivos y se alcanzaron aprendizajes significativos. Mediante la aplicación de una rúbrica de reflexión y auto-evaluación, diseñada especialmente para esta actividad, como podrá apreciarse en las gráficas que se presentan a continuación, la tendencia se ubica entre el 75 y $100 \%$ :

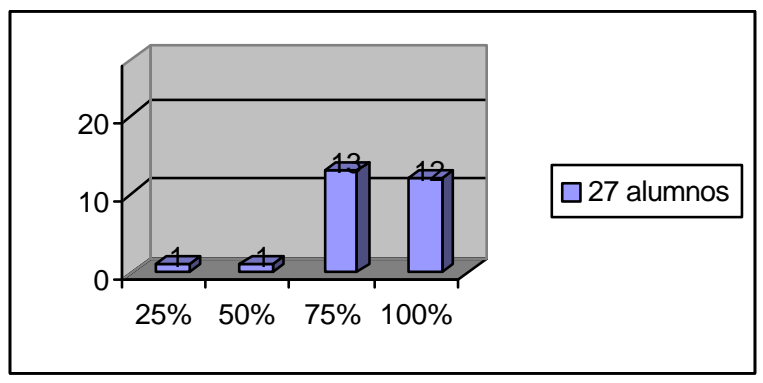

F.1b. Estuve atento a la explicación del maestro.

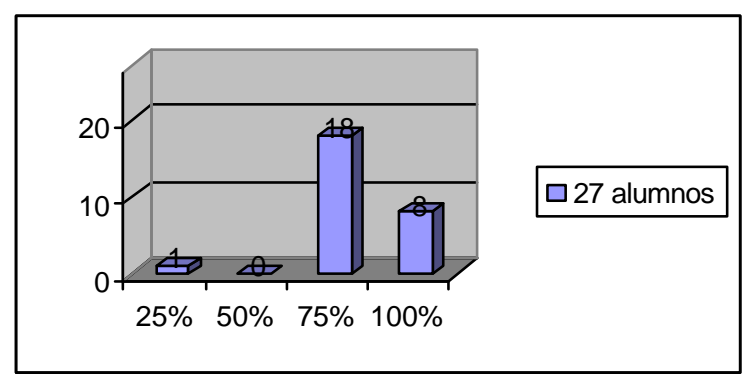

F.3b. ¿El tiempo asignado para cada parte de la tarea, fue optimizado por el grupo?

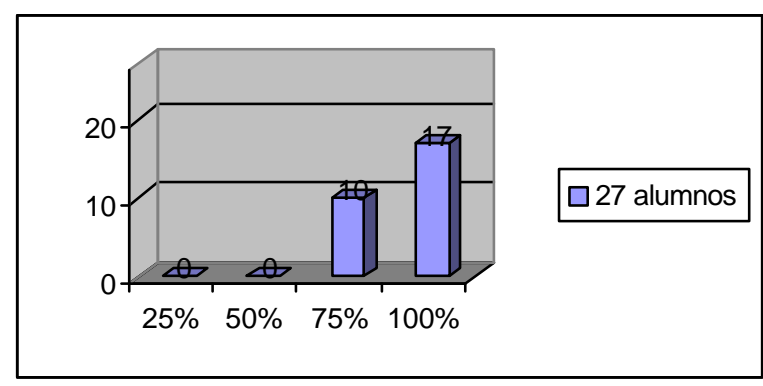

F.1b. ¿Cumpliste con el rol que se te asignó durante la realización de la tarea?

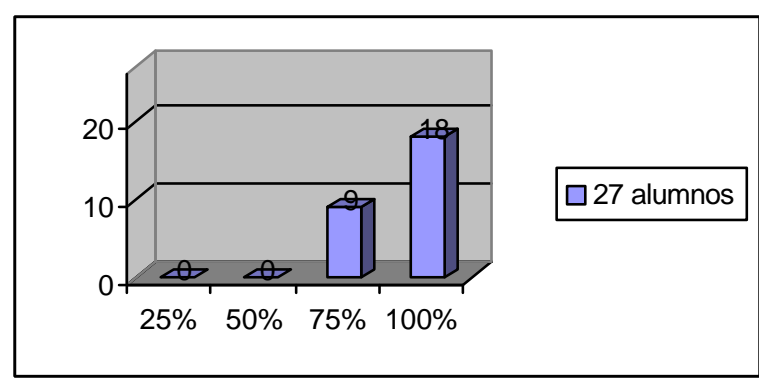

F.4b. ¿Los integrantes del grupo en su totalidad

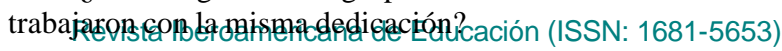




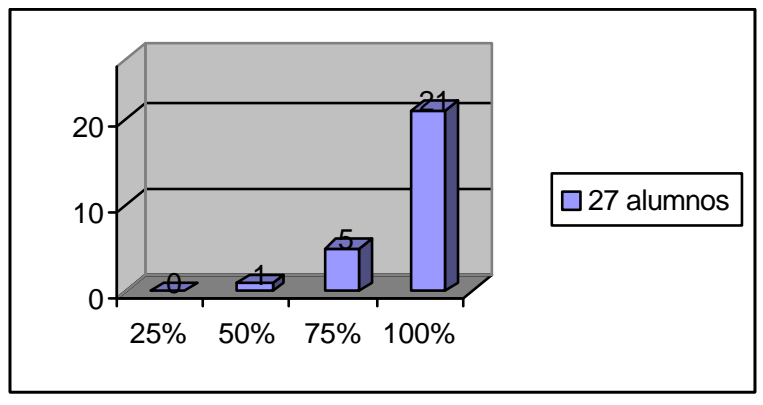

F.5b. ¿Se apoyaron lo suficiente al interior del grupo colaborativo. para que todos reforzaran los temas?

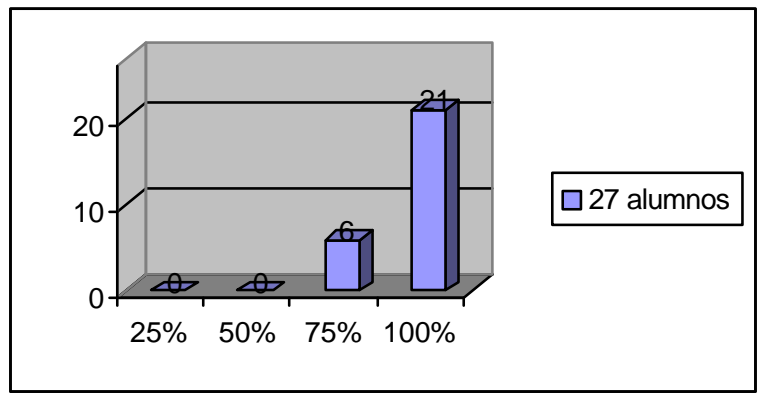

F.5b. ¿Se apoyaron lo suficiente al interior del grupo colaborativo, para que todos reforzaran los temas?

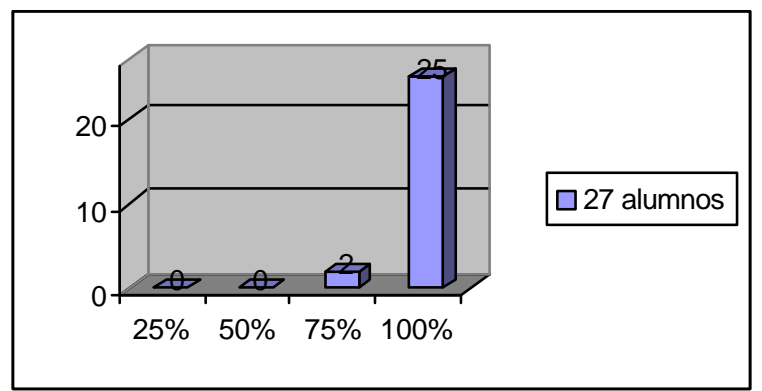

F.6b. ¿Lograron terminar la tarea con éxito?

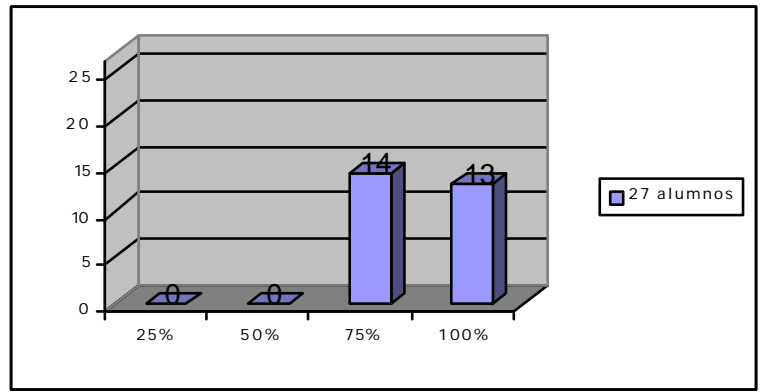

F. $8 \mathrm{~b}$ ¿Te cercioraste de que tus compañeros comprendieran la temática en estudio?

Para la actividad colaborativa virtual se plantearon los siguientes objetivos cognitivos:

- El alumno, conocerá, comparará, identificará los regímenes totalitarios y su ideología.

- Integrará nuevos conocimientos a los adquiridos durante el segundo parcial del semestre.

- Obtendrá la información en Biblioteca Digital.

Objetivos colaborativos:

- Compartir, información y enriquecer su conocimiento mediante el intercambio de los contenidos investigados, para complementar su conocimiento.

- Construir un cuadro de síntesis del totalitarismo, incluyendo: características relevantes, líderes principales, tendencias y valores que cultivaron.

- Al finalizar el cuadro de síntesis, cada grupo colaborativo, tuvo que realizar una propuesta de gobierno e ideología, en la cual se incluyeran los aspectos positivos de las estudiadas en esta actividad.

\section{RESULTADOS DE ACTIVIDAD COLABORATIVA VIRTUAL.}

Los grupos colaborativos se encontraron formados por 4 alumnos cada uno, mismos que podían conocer en la plataforma tecnológica de la Institución, lugar donde se integraron las instrucciones detalladas en todas sus fases. La información quedó concentrada en la misma plataforma en foros de discusión.

Los objetivos que planteados se lograron en su mayoría con porcentajes que van del 75 al $100 \%$, sin embargo como se muestra en las figuras, $5 \mathrm{c}, 7 \mathrm{c}$ y $8 \mathrm{c}$, en la modalidad virtual los alumnos percibieron 
que no se apoyaron, ni reforzaron el tema en estudio, tampoco valoraron el trabajo de sus compañeros así como no pudieron cerciorarse del aprendizaje de los mismos. Una posible respuesta a estos indicadores es que no tienen la suficiente práctica en este tipo de actividades y no han logrado establecer mejor comunicación virtual entre ellos.

Sea cualquiera el motivo, el profesor que implante este tipo de ejercicios, deberá tener presente el aplicar reforzadores verbales positivos en pro de la comunicación al interior de los grupos colaborativos. Los resultados se muestran a continuación en los gráficos realizados.

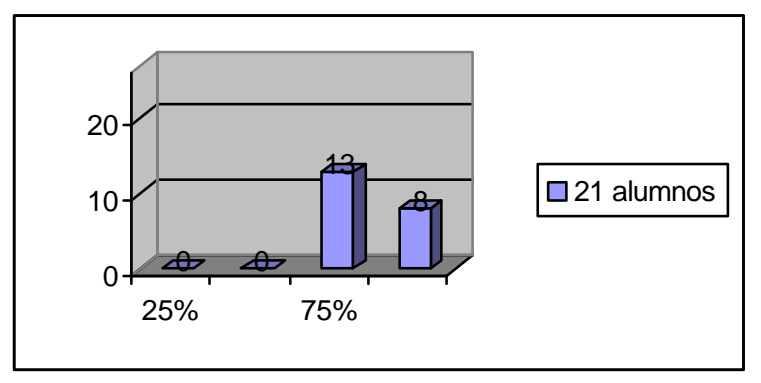

F.1c. Me comprometí con el grupo y con la meta fijada para esta actividad.

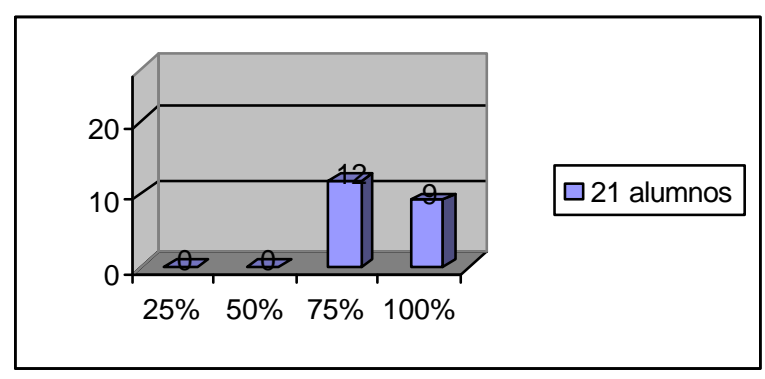

F.3c. ¿El grupo optimizó los tiempos?

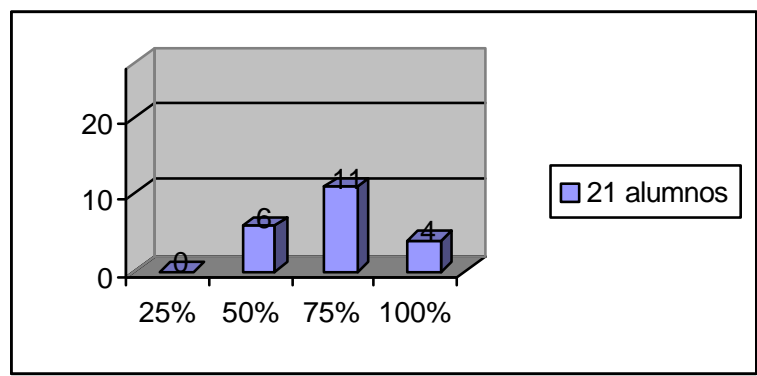

F.5c. ¿Se apoyaron entre los miembros del grupo de aprendizaje colaborativo, para lograr que todos reforzaran los temas?

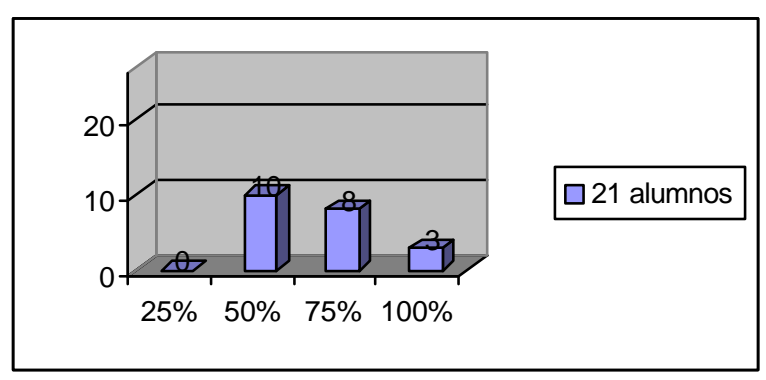

F.7c. ¿Valoraron el esfuerzo realizado por los miembros del grupo colaborativo?

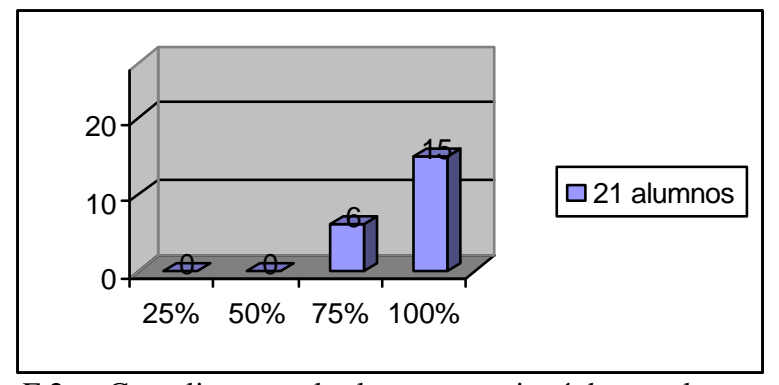

F.2c. ¿Cumpliste con el rol que se te asignó durante la realización de la tarea?

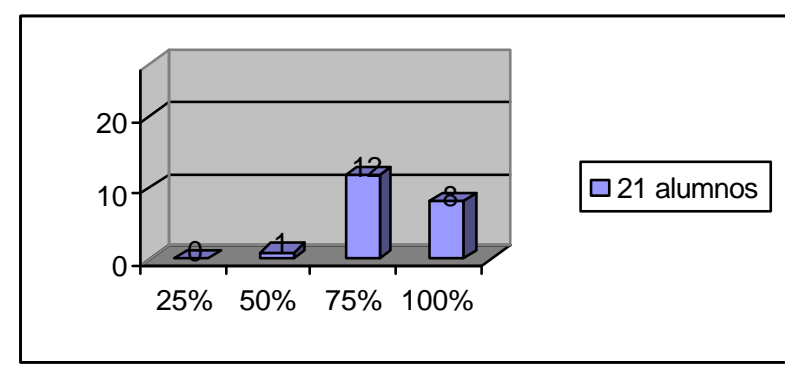

F.4c. ¿Los integrantes del grupo, trabajaron con la misma dedicación?

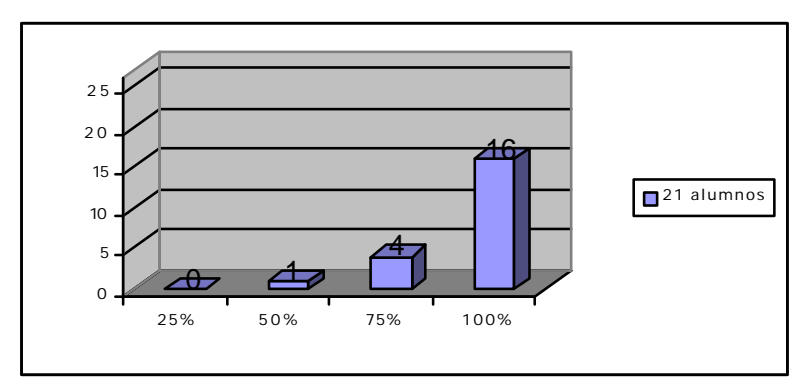

F.6c. ¿Lograron terminar la tarea con éxito?

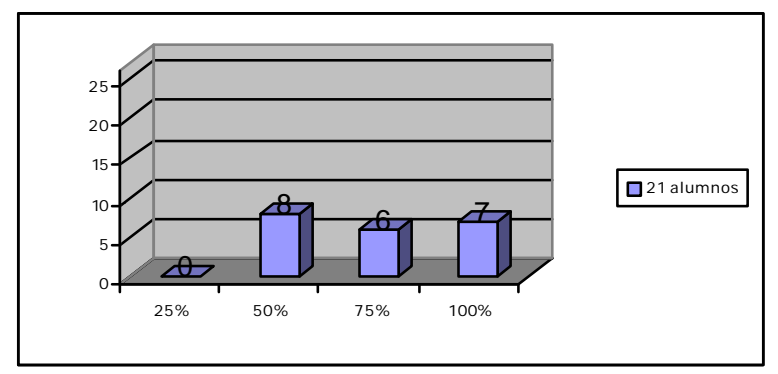

F.8c. ¿Te cercioraste, que tus compañeros comprendieran la temática en estudio?

Revista Iberoamericana de Educación (ISSN: 1681-5653) 


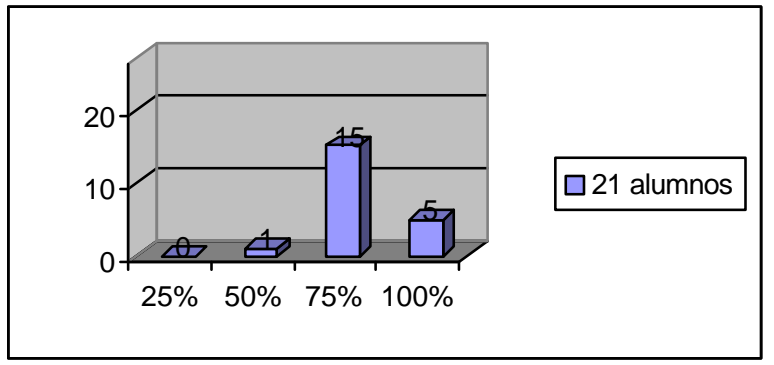

F.9c. ¿Los otros grupos cumplieron con los tiempos asignados, permitiendo que el trabajo que tu grupo realizaba evolucionara como se planeó??

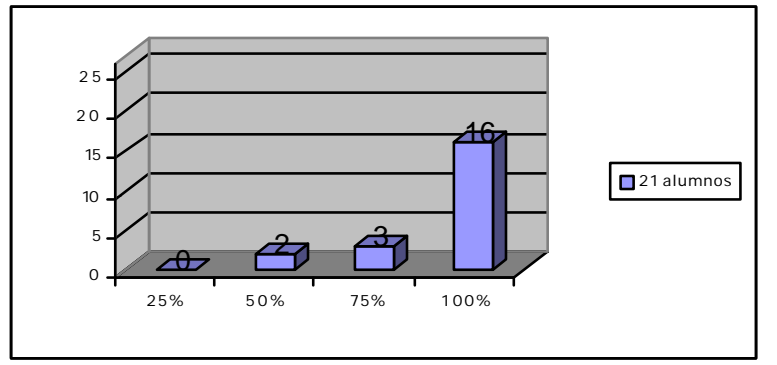

F.10c. ¿Crees conveniente realizar este tipo de actividades, como apoyo al desarrollo de tus habilidades sociales, intelectuales y cognitivas?

\section{REFLEXIONES A MANERA DE CONCLUSIÓN}

Uno de los principales retos en la actualidad es que los alumnos aprendan cómo aprender, que tengan plena conciencia del método o técnica que le favorece la adquisición del conocimiento, es decir, llegar al metaconocimiento.

Los grupos de aprendizaje colaborativo, proveen al alumno de habilidades que le ayudan a interactuar con sus pares, a la vez que le proporcionan destrezas para construir, descubrir, transformar y acrecentar los contenidos conceptuales; así como socializar en forma plena con las personas que se encuentran en su entorno. El intercambio de ideas, los análisis y discusiones que se dan al interior de un grupo de trabajo, enriquecen en mayor grado y menor lapso de tiempo, que cuando se intenta llegar a soluciones por si mismo.

La aplicación de las actividades colaborativas diseñadas en sus modalidades, informal, formal y virtual, descritas con anterioridad, en las que se incluyeron diferentes estrategias de aprendizaje, como fueron la construcción de un mapa mental, una $V$ heurística y un cuadro de síntesis, lograron no sólo la adquisición del conocimiento por los alumnos, sino que apoyaron el desarrollo de habilidades como el análisis, la síntesis, la evaluación, la comparación entre otras. Los resultados obtenidos por la adquisición de conocimientos fueron muy satisfactorios.

Las rúbricas diseñadas para evaluar las actividades realizadas, como se muestra en las gráficas anteriores, tienden hacia lo positivo, sin embargo es necesario seguir trabajando dentro del mismo modelo ya que hay rubros que deben ser reforzados. Tomando en cuenta que el éxito del grupo colaborativo, depende de todos los integrantes no de uno de sus miembros, los profesores tenemos que fortalecer las metas del trabajo grupal, es decir, hacer conciencia en los alumnos que no solo se trata de cumplir con una tarea, sino que todos deben dominar el contenido de la misma, igualmente preocuparse por que el conocimiento sea adquirido por los integrantes en su totalidad.

La debilidad que aún prevalece dentro de esta técnica, es la última mencionada, no se ha logrado que se apoyen completamente, puede ser que la edad que atraviesan los alumnos de la Prepa sea un factor importante, sin embargo deberemos seguir incidiendo en ese punto. De manera que al concluir su ciclo de estudios, todos los alumnos hayan adquirido el conocimiento en el mismo nivel, y sean los menos los que logren grados cobijados con el esfuerzo de otros.

Durante la aplicación y ejecución del trabajo colaborativo fue muy agradable para todos los que nos involucramos en el proceso, ya que formamos un verdadero grupo de aprendizaje. 


\section{BIBLIOGRAFÍA}

FUENTES, Norma, (2003). Trabajo colaborativo. Curso de capacitación ITESM.

MORENO, Bayardo Guadalupe. (1994). Didáctica, fundamentación y Práctica. México, Progreso.

VILLALOBOS, Pérez Cortés. (2002). Didáctica integrativa y el proceso de aprendizaje. México, Trillas.

JOHNSON, David W. JOHNSON, Roger T. JOHNSON, Holubec, E. (1995). Los Nuevos Círculos de Aprendizaje. EUA, ASCD. 


\title{
Contactar
}

Revista lberoamericana de Educación

\author{
Principal OEI
}

